compared with those admitted one year before the pandemics $(\mathrm{p}<0.001)$.

Conclusions This population-based study demonstrated the differential impacts of COVID-19 and SARS on rare disease patients in Hong Kong, a group that is currently not strategically protected. Real-time data analysis by age group within different populations could be of consideration when developing prioritisation guidelines in the future. Taken together, this study warrants cautious healthcare planning, with consideration of prioritising patients with rare diseases.

\section{TAKING THINKING TOGETHER FORWARD: A SCHEME LINKING PAEDIATRIC AND CAMHS TRAINEES}

Jo Cryer, Megan Fisher. UK

\subsection{6/bmjpo-2021-RCPCH.81}

Background The concept of joining up services and managing young people's mental health difficulties has never been more paramount. Covid has thrown us all into a world of uncertainty and this is exacerbated for young people with anxiety, underlying medical or psychiatric needs. At a time when linking people up to work and think together is the most needed, of course it is also the most challenging. Mental health presentations are an increasing part of the paediatric caseload and there is growing evidence that paediatric trainees feel ill-equipped to manage these patients. Equally CAMHS trainees reported they required greater access to seeing children under 5, assessing development and physical health. Only $33 \%$ of trainees we surveyed felt that their current training programme enabled them to achieve their curriculum requirements in paediatrics or mental health respectively.

Objectives We developed the concept of Thinking Together, to tackle this training gap. The scheme involves pairing paediatric and CAMHS trainees to share clinical encounters to foster a joint way of learning and working together, coming together to look at how we can link training to optimise patient care. CAMHS services are increasingly stretched, raising referral thresholds and making the need for closer working together across disciplines paramount.

Methods A pilot was launched in March 2016 where 30 trainees from CAMHS and Paediatrics were paired for a period of 6 months, attending at least two clinical encounters in each setting. Trainees were encouraged to explore a variety of learning possibilities, including clinics, referral meetings and signposting their partner to other relevant clinical opportunities.

In 2018 we extended the scheme in a second phase pilot to four centres across the UK. Feedback from participants involved was equally positive but there were logistical challenges. We reflected on feedback from the initial and second phase pilot and adapted the programme in 2019/2020. We aimed to consolidate the scheme in one centre and include workshops, which allowed focus on a topic relevant to both specialties, in addition to a forum for debrief discussions on challenging cases.

Results Prior to participating in Thinking Together, 70\% stated they had no experience of working in a jointly delivered paediatric/mental health clinic. $93 \%$ of participants felt that their patients benefitted from access to jointly delivered clinics. Feedback was very positive and identified that the scheme met training gaps for both specialties. Th adapted version of Thinking Together that we rolled out in 20192020 was also met with positive feedback and proved to be particularly beneficial during the pandemic, when trainee pairs could still participate in Thinking Together via virtual workshops.

Conclusions Covid has clearly brought challenges to every aspect of our lives but this is a training model that has been able to adapt in this unprecedented time, by means of virtual workshops and the enthusiasm of the participants to continue to pursue learning opportunities, including joining referral meetings remotely. Thinking Together has evolved into a successful model that we would like to embed in everyday practice.

\section{REDUCING NEONATAL INTENSIVE CARE ADMISSIONS OF TERM INFANTS DURING COVID-19}

Sina Durani, Lesley Alsford. UK

\subsection{6/bmjpo-2021-RCPCH.82}

Background Covid-19 is a novel disease which has had an impact on the delivery of health care services, including postnatal care. Term newborns may often require additional clinical input varying from postnatal wards to the neonatal intensive care unit.

Objectives To review the admissions of term neonates to a level 2 neonatal intensive care unit (NICU) and transitional care (TC) ward (a postnatal ward) during the COVID-19 period.

Methods A retrospective audit was carried out of term neonatal admissions to NICU and TC during 1st March 2019-31st August 2019 (Period 1) and compared with 1st March 2020 $31^{\text {st }}$ August 2020 (period 2; Covid - 19 period). Babies born at term $(>37 / 40)$ were included. Babies who were premature $(<37 / 40)$ or repatriated from other hospitals were excluded. This also included reviewing term babies re-admitted from home within 7 days of birth.

Results Admissions to NICU had reduced by $1 / 3$ (143 during period 1, 97 during period 2). Similarly, there was a marked reduction in admissions related to respiratory distress (64 in period 1, 42 in period 2) and infection (14 in period 1, 4 in period 2). There were only 2 direct admissions due to Covid19. There were similar numbers of admissions from home (31 in period 1, 35 in period 2). There was an increase in the number of babies admitted from D4-5 of life, yet NICU admissions from home had nearly halved (21 in Period 1, 11 in period 2). There was an increase in admissions to the TC ward instead (10 in Period 1, 24 in Period 2), including more babies requiring treatment for jaundice. The admissions to the TC ward were mostly not in line with the existing 'readmission from home policy' however there were no complications from these admissions. There was a reduction in the readmissions from home due to hypernatremic dehydration and infection.

Conclusions Although overall delivery numbers were similar between the two time periods, Elective C-sections occurred off site which may explain the reduction in admissions for respiratory distress. The improvement in isolation and infection protocols could explain the reduction in admissions due 